\title{
Fast and Straightforward Synthesis of Luminescent Titanium(IV) Dioxide Quantum Dots
}

\author{
Václav Štengl, ${ }^{1}$ Jiří Henych, ${ }^{1}$ Martin Št'astný, ${ }^{1}$ and Martin Kormunda ${ }^{2}$ \\ ${ }^{1}$ Department of Material Chemistry, Institute of Inorganic Chemistry AS CR v.v.i., 25068 Řež, Czech Republic \\ ${ }^{2}$ Department of Physics, Faculty of Science, J. E. Purkyně University in Ústí nad Labem, 40096 Ustí nad Labem, Czech Republic \\ Correspondence should be addressed to Václav Štengl; stengl@iic.cas.cz
}

Received 20 April 2017; Accepted 15 June 2017; Published 20 July 2017

Academic Editor: Martin Andersson

Copyright @ 2017 Václav Štengl et al. This is an open access article distributed under the Creative Commons Attribution License, which permits unrestricted use, distribution, and reproduction in any medium, provided the original work is properly cited.

\begin{abstract}
The nucleus of titania was prepared by reaction of solution titanium oxosulphate with hydrazine hydrate. These titania nuclei were used for titania quantum dots synthesis by a simple and fast method. The prepared titanium(IV) dioxide quantum dots were characterized by measurement of X-ray powder diffraction (XRD), X-ray photoelectron spectroscopy (XPS), atomic force microscopy (AFM), high-resolution electron microscopy (HRTEM), and selected area electron diffraction (SAED). The optical properties were determined by photoluminescence $(\mathrm{PL})$ spectra. The prepared titanium(IV) dioxide quantum dots have the narrow range of UV excitation (365-400 nm) and also a close range of emission maxima (450-500 $\mathrm{nm})$.
\end{abstract}

\section{Introduction}

Titan is one of the most abundant metals of Earth's crust. The most stable and therefore most widely used titanium compounds are the oxides, in particular titanium(IV) dioxide $\mathrm{TiO} 2$. In practice, the most commonly used powder rutile is titanium white, and these fine white powders are among the best available white pigments. $\mathrm{TiO} 2$ in powder form strongly scatters light and has a high opacity. It is therefore used as its pigments provide whiteness, and opacity of paints, coatings, plastics, paper, inks, food, cosmetics, and pharmaceuticals, for example, tablets or toothpaste. It is used as a food white dye E171. In cosmetics, it is added to the creams as a pigment and a thickener and as a dye it is also used in tattoos. With its high index of refraction, opacity, UV resistance, and stability it is frequently used as sunscreen ingredient. Against sunscreen based on chemical absorbers, create sunscreens containing physical absorbers, titanium dioxide, and zinc oxide, often less irritated and allergic skin reactions. In medicine, it enables growth of bones with the implants such as artificial joints and teeth. Quantum dots have remarkable advantages in bioimaging, biosensing, and other biomedical applications. Commercially prepared quantum dots based on Cd sulfides or selenides without surface treatment for their toxicity are not suitable for biological applications. The toxicity and biosafety materials of carbon nanostructures such as carbon nanotubes, graphene, graphene oxide $[1,2]$, carbon, graphene quantum dots [3], iron oxide, and titanium oxide [4] are intensively under study.

The electronic structure of rutile quantum dots is investigated via the first-principles band structure method. First, propose a model to passivate the rutile titania surfaces for the local density approximation calculations. In this example, pseudo hydrogen atoms are used to passivate the surface dangling bonds, which remove the localized in-gap surface states in the titania quantum dots [5]. Self-ordered titania quantum dot array was prepared by the process of titanium anodic oxidation through a nanoporous alumina template on a silicon substrate [6]. Titania quantum dots on graphene nanosheets in water-oil suspension display high performance as an anode material for lithium-ion batteries [7].

Nonluminescent titania quantum dots were prepared for photocatalytic degradation methyl orange and methylene blue [8], carmine dye [9], and quantum dots interface in the recombination processes of quantum dot sensitized solar cells [10].

The top-down method was used for the preparation of quantum dots from natural minerals (graphite, molybdenite, 


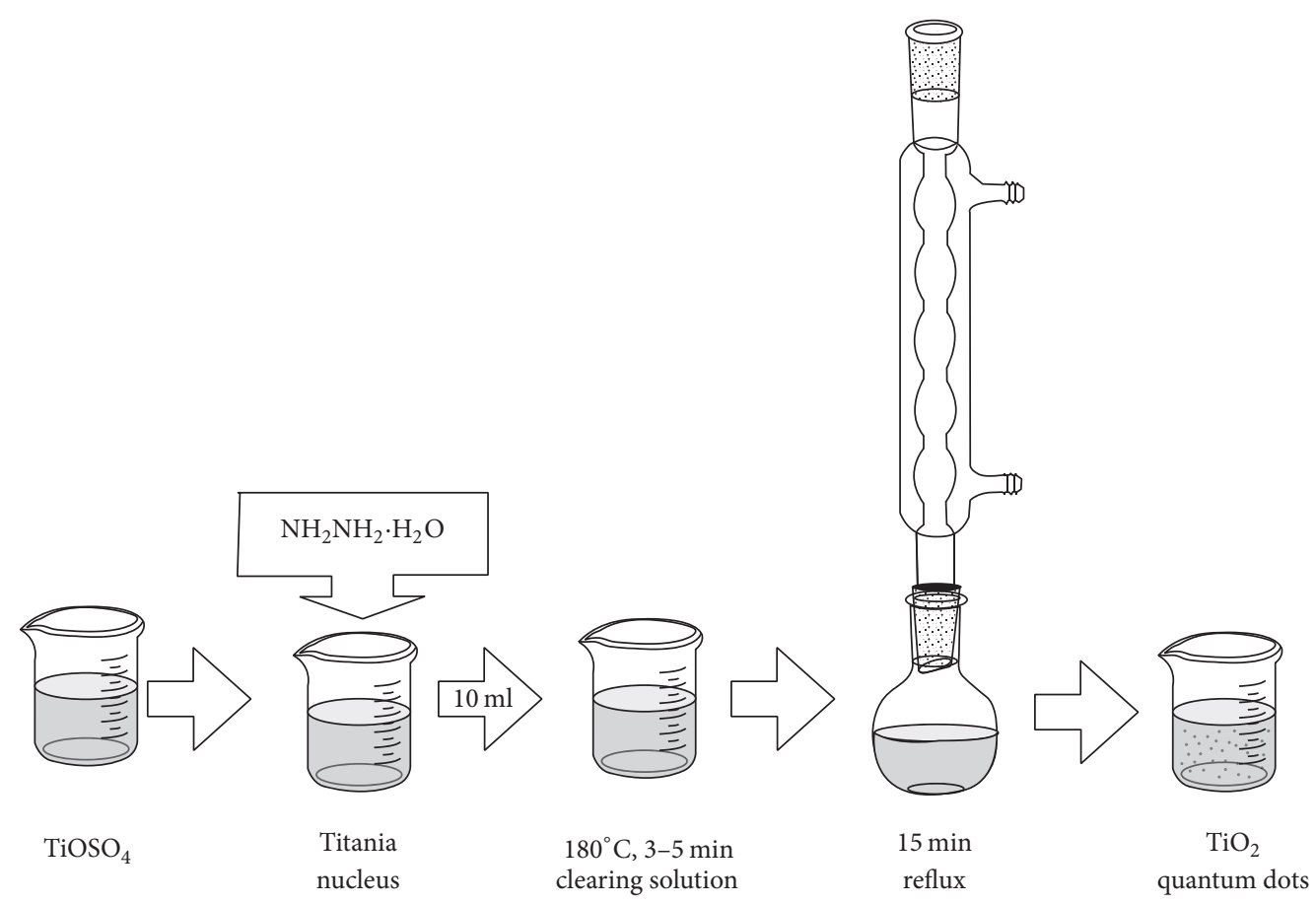

FIgURE 1: Schematic of preparation of $\mathrm{TiO}_{2}$ quantum dots using reflux.

and tungstenite) and synthetic layered materials (h-BN, h$\mathrm{BCN}$, and g-C3N4). The bulk sample was exfoliated using high intensity ultrasound in the pressurized reactor and obtained nanosheets form with ethylene glycol under reflux at temperature $198^{\circ} \mathrm{C}$ quantum dots [11-13].

In this paper, we for the first time presented fast, simple, and scalable bottom-up method for synthesis luminescent titania quantum dots TiQDTs from titania oxosulphate and hydrazine hydrate as coupling agents and ethylene glycol, dimethyl sulfoxide, octadecene, dodecanethiol, dimethylformamide, and tetraethyl orthosilicate as solvents. The TiQDTs thus formed are highly luminescent, with the emission maxima controlled by the used excitation wavelength.

\section{Experimental}

2.1. Titania Quantum Dots Synthesis. All used chemicals, titanium oxosulphate TiOSO4, hydrazine hydrate $\mathrm{NH} 2 \mathrm{NH} 2 \oplus \mathrm{H} 2 \mathrm{O}$, ethylene glycol $\mathrm{CH} 2 \mathrm{CH} 2(\mathrm{OH}) 2$, dimethyl sulfoxide (DMSO) (CH3)2SO, octadecene $\mathrm{C} 18 \mathrm{H} 36$, dodecanethiol $\mathrm{CH} 3(\mathrm{CH} 2) 10 \mathrm{CH} 2 \mathrm{SH}$, dimethylformamide $\mathrm{COHN}(\mathrm{CH} 3) 2$, and tetraethyl orthosilicate (TEOS) $\mathrm{Si}(\mathrm{OC} 2 \mathrm{H} 5) 4$, were obtained from Sigma-Aldrich.

$100 \mathrm{ml} 0.3 \mathrm{M}$ solution of TiOSO 4 was mixed with $20 \mathrm{ml}$ $80 \%$ hydrazine hydrate, and the milk-like solution is formed; the $10 \mathrm{ml}$ of this solution was added to $100 \mathrm{ml}$ of ethylene glycol and the reaction mixture was heated under stirring at $180^{\circ} \mathrm{C}$. For 3-5 minutes, the reaction mixture turned bright and throughout the next 15 minutes UV luminescence was originated. The reaction solution was allowed to cool to room temperature and centrifuged at $6000 \mathrm{rpm}$. The same experiment was performed with dimethyl sulfoxide, octadecene, dodecanethiol, dimethylformamide, and tetraethyl orthosilicate instead of ethylene glycol. The schematic of synthesis $\mathrm{TiO} 2$ quantum dots is presented in Figure 1.

2.2. Characterization Methods. Diffraction patterns were collected with diffractometer Bruker D2 equipped with conventional X-ray tube ( $\mathrm{Cu} \mathrm{K} \alpha$ radiation, $30 \mathrm{kV}, 10 \mathrm{~mA})$. Qualitative analysis was performed with the DiffracPlus Eva software package (Bruker AXS, Germany) using the JCPDS PDF-2 database [14].

The morphology of the titania quantum dots was inspected by transmission electron microscopy (TEM) using a $300 \mathrm{kV}$ TEM microscope JEOL 3010F. As a specimen support for TEM investigations, a microscopic copper grid covered by a thin transparent carbon film was used.

High-resolution transmission electron microscope (HRTEM) analysis was performed on FEI Talos F200X. The microscope is equipped with Super-X EDS system with four silicon drift detectors (SDDs) for precise and quantitative energy dispersive X-ray (EDS) analysis for advanced materials characterization. The 300-Mesh Regular Copper Grid coated by Silicon Dioxide ( $\mathrm{SiO} 2) /$ Monoxide $(\mathrm{SiO})$ was used for sample preparation.

AFM images were obtained using a Bruker Dimension ICON FastScan microscope. AFM samples were prepared by a spin-coating method that consisted of pipetting an aqueous suspension of h-BN-TiO2 samples onto an atomically smooth support of synthetic mica. The suspension was spin coated on the mica substrate at 6000 r.p.m. For atomic force microscopy (AFM) analysis, a silicon tip on a nitride lever was used with ScanAsyst, air contact mode (resonance frequencies $50 / 90 \mathrm{kHz})$. 
The hydrodynamic diameter and size distribution of the quantum dots were determined by dynamic light scattering using light scattering using a ZEN 1600 (Malvern Co., USA) particle size analyzer.

XPS measurements were performed with the hemispherical analyzer Phoibos 100 (Specs GmbH, Germany) at takeoff angle of $90 \mathrm{deg}$ and the spectra were referenced to the peak of aliphatic C-C bonds at $284.5 \mathrm{eV}$. The XR-50 (Specs $\mathrm{GmbH}$, Germany) nonmonochromatic X-ray source with spectral line $\mathrm{Al} \mathrm{Ka}$ (photon energy $1486.6 \mathrm{eV}$ ) and spectral line $\mathrm{Mg} \mathrm{Ka}$ (photon energy $1253.6 \mathrm{eV}$ ) was used to induce the electron emission. The elemental composition was calculated from survey spectra taken at pass energy $40 \mathrm{eV}$. The electron flood gun was not used for charge compensation. Ti $2 p$ high-resolution spectra taken at pass energy $10 \mathrm{eV}$ were constructed from 30 measurements to obtain the information on the bonding of Ti. The program CasaXPS was utilized for the computer processing of the spectra. Relative sensitivity factor was used from built-in CasaXPS database. The Shirley background shape was used, and the components used for the peak deconvolution were mixed Gauss-Lorentzian lines (70\% Gaussian and 30\% Lorentzian). The ethylene glycol solution of titania quantum dots was dropped on Si wafer and dried in a vacuum chamber for 30 minutes. The residuum was visible on the wafer and immediately inserted into XPS system.

The PL and UV-vis spectra of the titania quantum dots were determined by Avantes AvaSpec 2048 equipped with high-power (5 W) LEDs at wavelengths $365,375,385,395$, and $405 \mathrm{~nm}$ as excitation sources.

\section{Results and Discussion}

Titanyl oxosulphate TiOSO4 reacts with hydrazine hydrate and nanosized nucleus titania precursor from titanium oxide and hydrazine sulphate are formed:

$$
\mathrm{TiOSO} 4+\mathrm{N} 2 \mathrm{H} 6 \mathrm{O} \Longrightarrow \mathrm{TiO} 2+\mathrm{N} 2 \mathrm{H} 6 \mathrm{SO} 4
$$

The particle size of titania precursor measured using dynamic laser scattering is showed in Figure 2; the determined average size was $<2 \mathrm{~nm}$. The white titania precursor reacts with organic solvents molecules and leads to its encapsulation (micelle formation) and the reaction solution was gradually clarified. After initial encapsulation, remaining molecules of organic solvents form connections between the individual micelles to form a network within the aqueous media. The resulting hydrogel forms a diffusion and a relatively constant concentration of the encapsulated particles in the gel.

The XRD pattern of purred TiQDTs using dialysis membrane from ethylene glycol is presented in Figure 3. Only the diffraction peaks of anatase PDF 21-1272 were detected. Extending the diffraction bands and increased background is caused by the poor crystallinity of the sample.

Titanium oxides are widely reported in literature, when titanium oxides can exist in various stoichiometry and at different binding energies (BE) of $\mathrm{Ti} 2 \mathrm{p} 3 / 2$ for $\mathrm{TiO} 2$ at

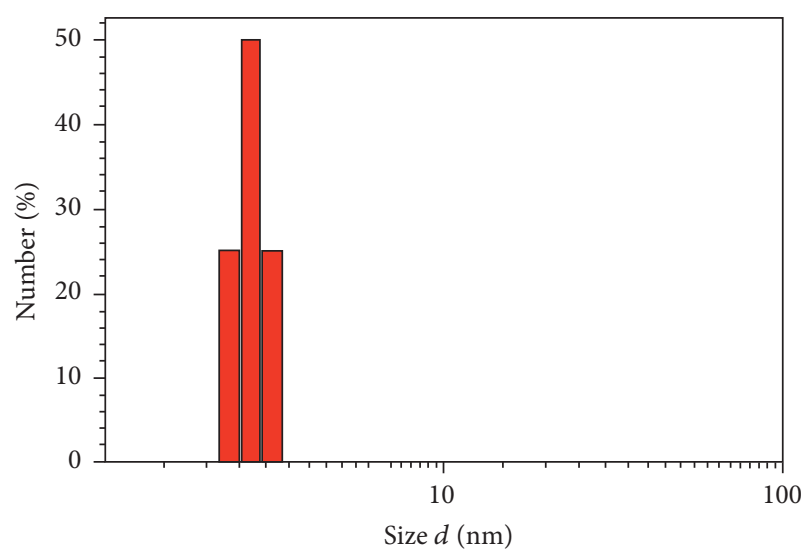

FIGURe 2: Dynamic laser scattering of nucleus titanium(IV) dioxide precursor.

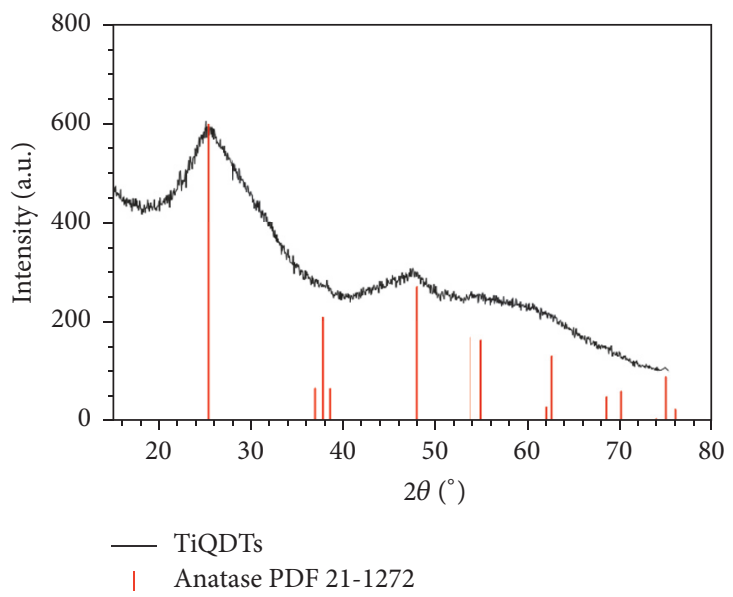

Figure 3: XRD pattern of purified TiQDTs.

$459.1 \mathrm{eV}, \mathrm{Ti} 3 \mathrm{O} 5$ at $457.9 \mathrm{eV}, \mathrm{Ti} 2 \mathrm{O} 3$ at $456.7 \mathrm{eV}, \mathrm{TiO}$ at $455.1 \mathrm{eV}$, and last metal $\mathrm{Ti}$ at $453.9[15,16]$. Each Ti oxide is also a component in $\mathrm{O}$ 1s but the reliable literature data are rather poor for some of the oxides. The elsewhere reported shift in Ti $2 p$ between Ti4+ and Ti3+ is from $1.2 \mathrm{eV}$ [16] up to $1.6 \mathrm{eV}[17,18]$ and between Ti4+ and Ti2+ from $2.4 \mathrm{eV}$ [12] up to $3.1 \mathrm{eV}$ [15]. The reported $\mathrm{BE}$ of components varies in the literature due to the calibration process and the charging effect. There are also reported binding energies of $\mathrm{Ti} 2 \mathrm{p} 3 / 2$ and also $\mathrm{O}$ 1s for $\mathrm{TiO} 2$ at $458.5 \mathrm{eV}-530 \mathrm{eV}$, Ti2 3 at about $455.6 \mathrm{eV}-531.5 \mathrm{eV}$, and $\mathrm{TiO}$ at $454.3 \mathrm{eV}-531.4 \mathrm{eV}$ [16]. The $\mathrm{BE}$ of $\mathrm{Ti} 2 \mathrm{p} 3 / 2$ for $\mathrm{TiO} 2$ is commonly referred to values about $459 \mathrm{eV}$ [17-19] and corresponding to $\mathrm{O} 1 \mathrm{~s}$ to values about $530 \mathrm{eV}$ [20] or $531 \mathrm{eV}$ [18]. Photoelectrons from Ti 2p are spin splinted to Ti $2 \mathrm{p} 3 / 2$ and Ti $2 \mathrm{p} 1 / 2$ separated about $5.73 \mathrm{eV}$ [17].

The basic composition of the sample was calculated from survey spectra taken by Mg source (see Figure 4(a)) because $\mathrm{Al}$ source induced Auger's electrons not related to Ti atoms from the sample with kinetic energy about $1025 \mathrm{eV}$ which is also kinetic energy of photoelectrons from $\mathrm{Ti} 2 \mathrm{p}$; see 


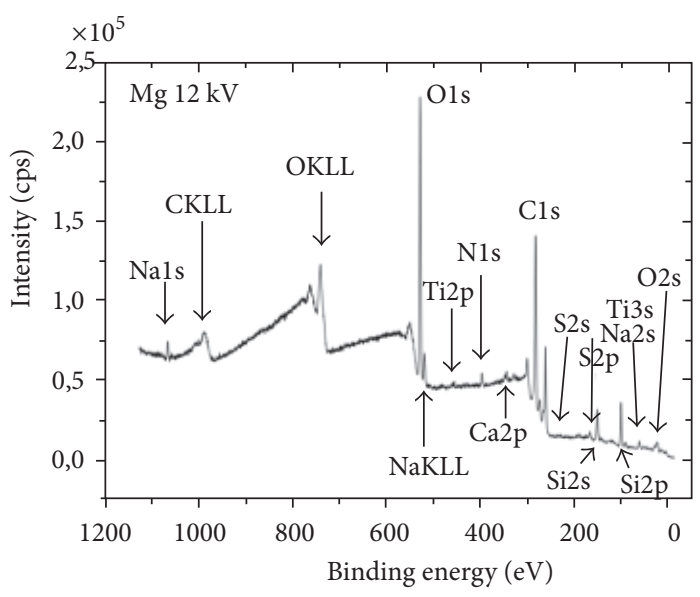

(a)

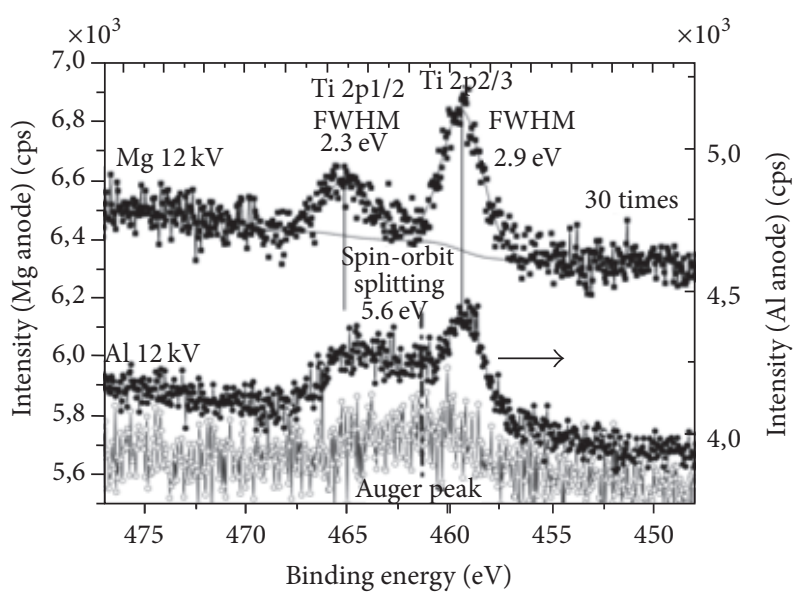

(b)

FIGURE 4: (a) XPS survey spectra of titanium oxide quantum dots with Mg anode. (b) XPS high-resolution spectra of Ti $2 \mathrm{p}$ region with indicated spin-orbit split and FWHM values for each orbital taken with $\mathrm{Mg}$ anode (full square) and spectra taken with $\mathrm{Al}$ anode on the same sample (full circle) and taken with $\mathrm{Al}$ anode on diluted sample (Ti at not detectable level) for Auger peak confirmation (open circle).

in Figure 4(b), full circles. It was confirmed on the sample with Ti $2 p$ under detection limit (diluted sample); see in Figure 4(b), open circles. The basic sample composition in [at.\%] is $\mathrm{C}=55.1$ (RSF 1), $\mathrm{Ca}=0.5(\mathrm{RSF} 5.13), \mathrm{N}=2.0$ (RSF 1.77), $\mathrm{Na}=0.5$ (RSF 7.99), $\mathrm{O}=26.8$ (RSF 2.85), $\mathrm{S}=1.4(\mathrm{RSF} 1.74), \mathrm{Si}=13.6(\mathrm{RSF} 0.865)$, and $\mathrm{Ti}=0.3$ (RSF 7.9). The silicon is probably the wafer used in sample preparation and oxygen content is influenced by native $\mathrm{SiO} 2$ on the wafer surface. The titanium content is rather small, but after data accumulation for 30 times it is well defined and shown in Figure 4(b). The relative sensitivity of XPS to $\mathrm{Ti}$ (Ti $2 \mathrm{p}$ orbitals) is given by RSF, listed above, and the value for Ti $2 p$ is one of the highest from the detected elements; moreover the presence of $\mathrm{Ti}$ in the sample was confirmed by XRD measurements. A possible misinterpretation of Ti $2 \mathrm{p}$ with an Auger line was avoided by comparison between spectra obtained at two different excitation energies.

Moreover the Ti $2 \mathrm{p}$ spectra were successfully deconvoluted to the single peaks representing Ti2p $3 / 2$ and Ti2p1/2 with the spin-orbital split about $5.6 \mathrm{eV}$, about $3 \%$ less than expected, and the fit keeps the theoretical $3: 2$ ratio of peak areas. It confirms that these two peaks are $\mathrm{p}$ orbitals and in this part of spectrum it can be only Ti $2 p$. The other element in close distance is $\mathrm{Ru} 3 \mathrm{p} 3 / 2$ at $461 \mathrm{eV}$ and $\mathrm{Ru} 3 \mathrm{pl} / 2$ at $483 \mathrm{eV}$ but it cannot explain peak at $466 \mathrm{eV}$ belonging to $\mathrm{Ti} 2 \mathrm{pl} / 2$. The FWHM values indicated in Figure 4(b) are rather bigger, but it can be the influence of data accumulation process. Therefore we conclude that the titanium is present in XPS detectable level and the binding energy of Ti2p $3 / 2$ about $459 \mathrm{eV}$ indicates dominant presence of $\mathrm{TiO} 2$ state.

Figure 5 shows the typical particle size distribution of synthesized titania quantum dots in dimethyl sulfoxide, octadecene, dodecanethiol, dimethylformamide, tetraethyl orthosilicate, and ethylene glycol measured by dynamic laser scattering. The size of the particles as determined by laser scattering is considerably larger than that measured by TEM. The laser scattering gives a mean hydrodynamic diameter of

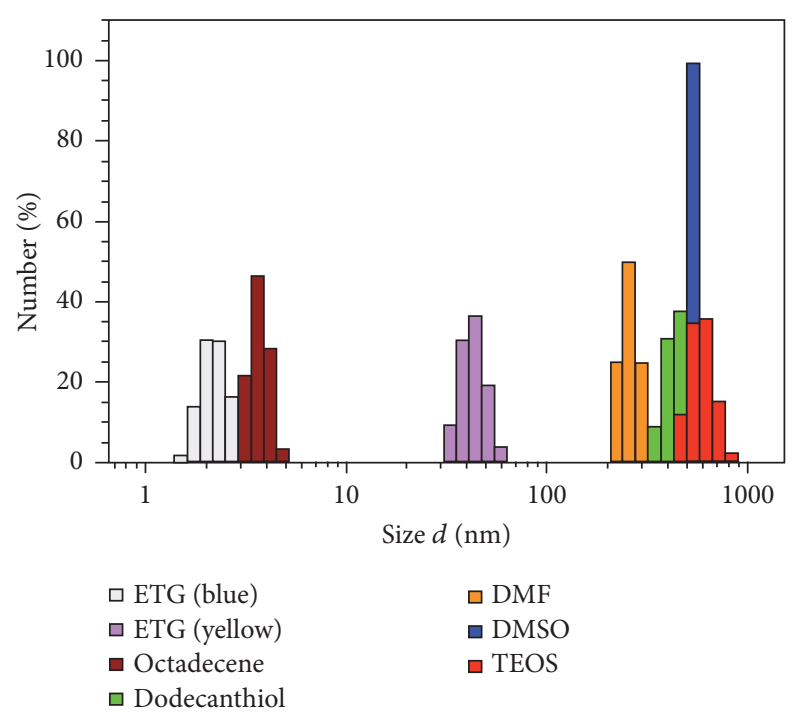

FIGURE 5: Hydrodynamic diameter of prepared TiQDTs for different solvating agents.

$\mathrm{TiO} 2$ core surrounded by the organic solvating layers, which is influenced by viscosity and concentration of the solution. In other words, TEM analysis determines the size of the core quantum dots. Hydrodynamic diameter increases depending on the length of the carbon chain with several molecular sizes of a solvating agent.

Image processing analysis (ImageJ software) of HRTEM micrograph was used to achieve a refinement of microstructure in the sense that we can more accurately analyze crystalline and amorphous domains and the boundaries between them. Also, from Fourier transform (FFT) it is possible to determine and to index crystallographic planes and find their orientations. Figure 6 shows HRTEM micrograph of anatase quantum dots prepared in ethylene glycol. The area used for 


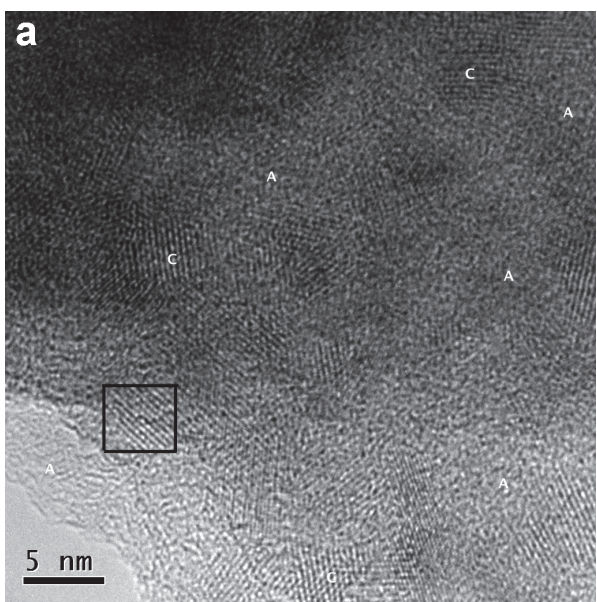

(a)

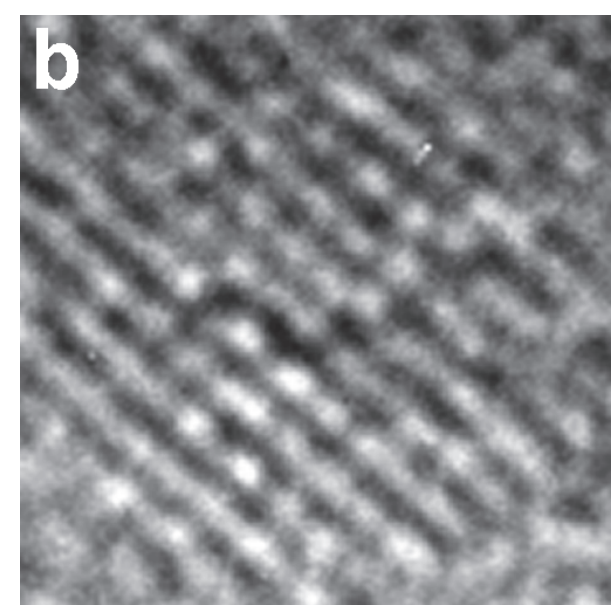

(b)

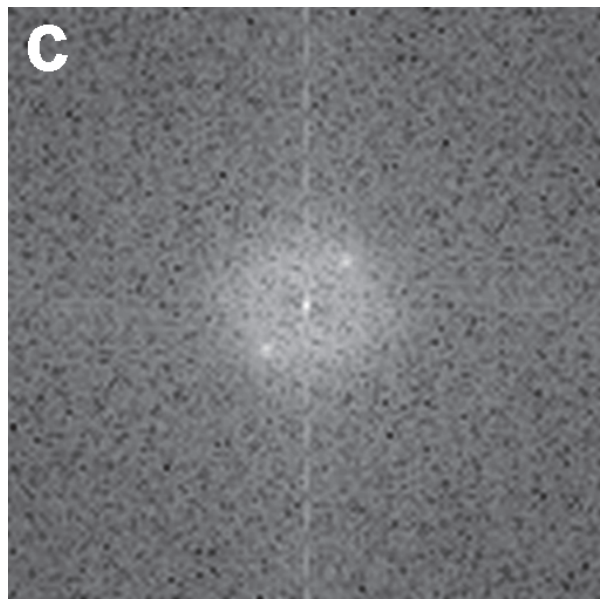

(c)

FIGURE 6: (a) HRTEM image of anatase quantum dots. The area used for image processing is marked with a black square. A: amorphous region. C: crystalline area. (b) Selected area and (c) Fourier transform of selected area.

image processing is marked with a black square indicating amorphous region (domains) and $\mathrm{C}$ denoted crystalline area. Figure 6(b) shows the region of the original image taken with mask of $512 \times 512$ pixels; Fourier transform of this area is in Figure 6(c) giving the interlayer spacing to $0.35 \mathrm{~nm}$ corresponding to (101) crystal plane of anatase (PDF 211272). Considering precise HRTEM investigation, we found a small fraction of amorphous materials. Crystalline anatase quantum dots are with some amorphous domains with the average diameter of 5-6 $\mathrm{nm}$ and are interlayered. Figure 7 presented Selected Area Diffraction Patterns (SAED) of prepared quantum dots analyzed by program ProcessDiffraction [21], the diffraction patterns well corresponding with anatase $\mathrm{TiO} 2$ (PDF 21-1272).

For HRTEM investigation is important to sample preparation, and remains of DMSO were well washed from TEM grid. In the TEM Figure 8(a) see solitary quantum dots of large size of about $4 \mathrm{~nm}$. The HRTEM investigation in Figure 8(b) shows the darker cover and crystalline core; the FFT analysis determines the interlayer spacing $0.19 \mathrm{~nm}$. Corresponding $d_{\mathrm{hkl}}=0.189 \mathrm{~nm}$ (200) for anatase has intensity

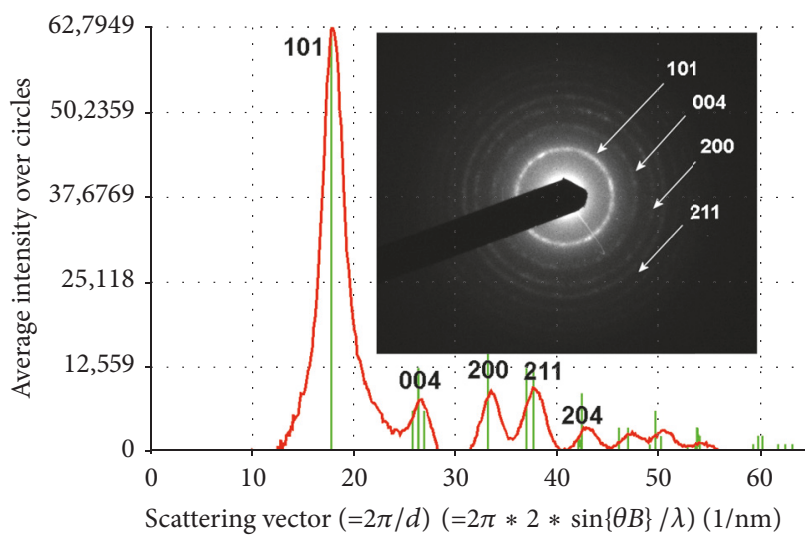

FIGURE 7: SAED pattern of TiQDTs analyzed by ProcessDifraction software.

$\sim 35$ a.u. and for carbon about $d_{\mathrm{hkl}} \sim 0.19 \mathrm{~nm}$ has intensity $<10$ a.u. Based on hkl intensities it is possible to assign this interlayer spacing to anatase and then carbon; origin 


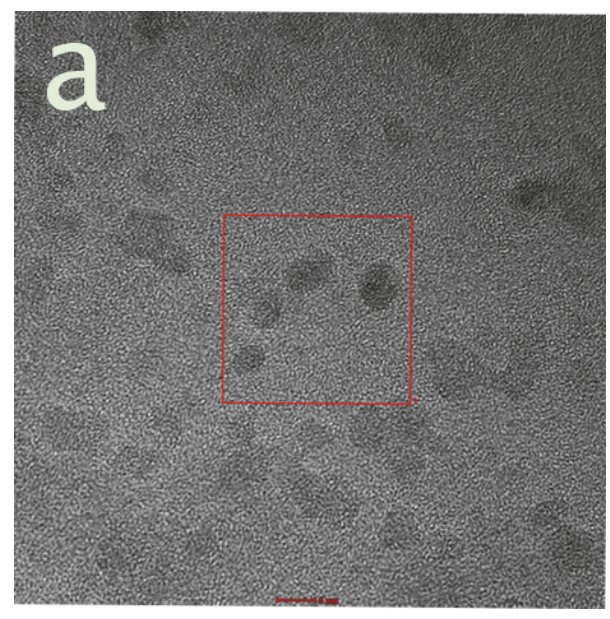

(a)

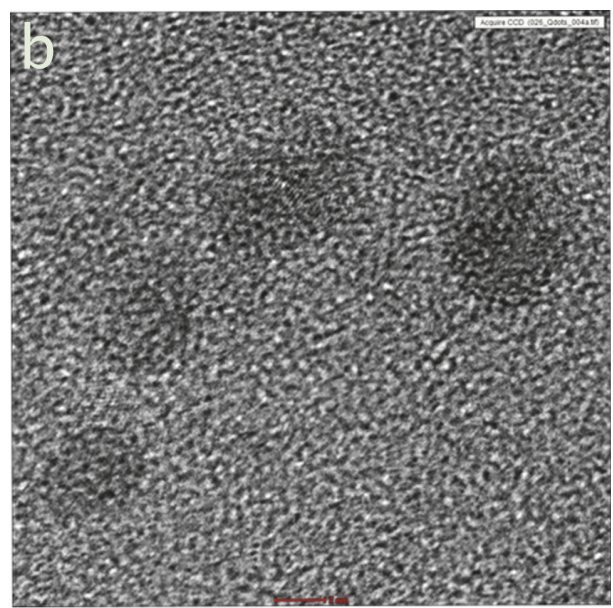

(b)

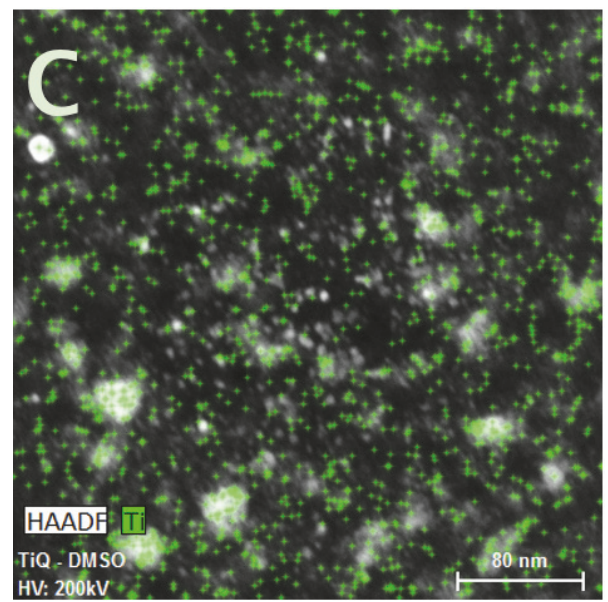

(c)

FIgURE 8: Titania quantum dots prepared in DMSO. (a) TEM images, magnification 600,000x, (b) HRTEM images, magnification $2,000,000 \mathrm{x}$, and (c) STEM images of mapping of elements, Ti green dot.

of carbon with DMSO carbonization is not probable [22]. Mapping of elements of the same sample solution, where atoms $\mathrm{Ti}$ are displayed green, is in Figure 8(c).

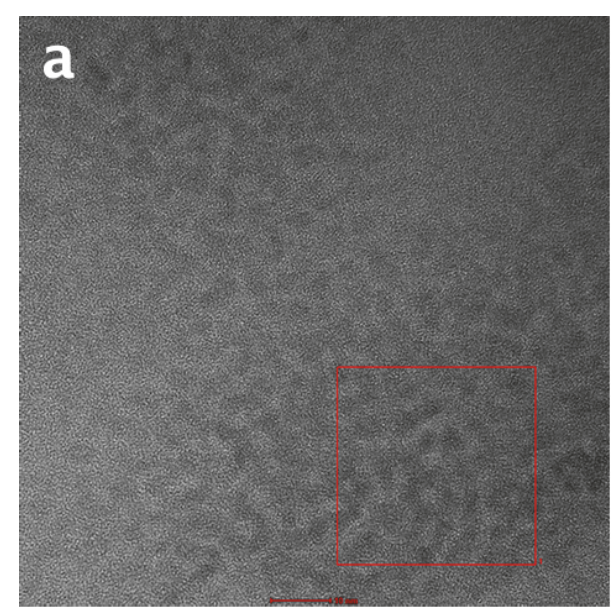

(a)

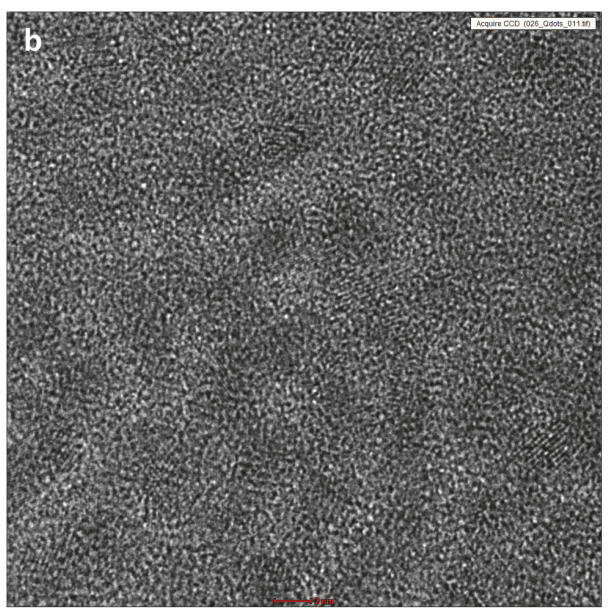

(b)

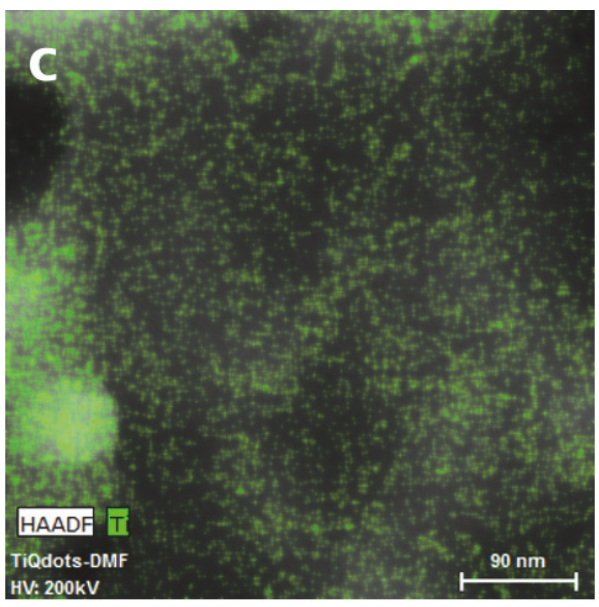

(c)

FIgURE 9: Titania quantum dots prepared in DMF. (a) TEM images, magnification 600,000x, (b) HRTEM images, magnification 2,000,000x, and (c) STEM images of mapping of elements, Ti green dot.

In the next images, Figure 9 is TEM images of titania quantum dots prepared in dimethylformamide solution. The HRTEM analysis in Figure 9(b) consists again of amorphous 

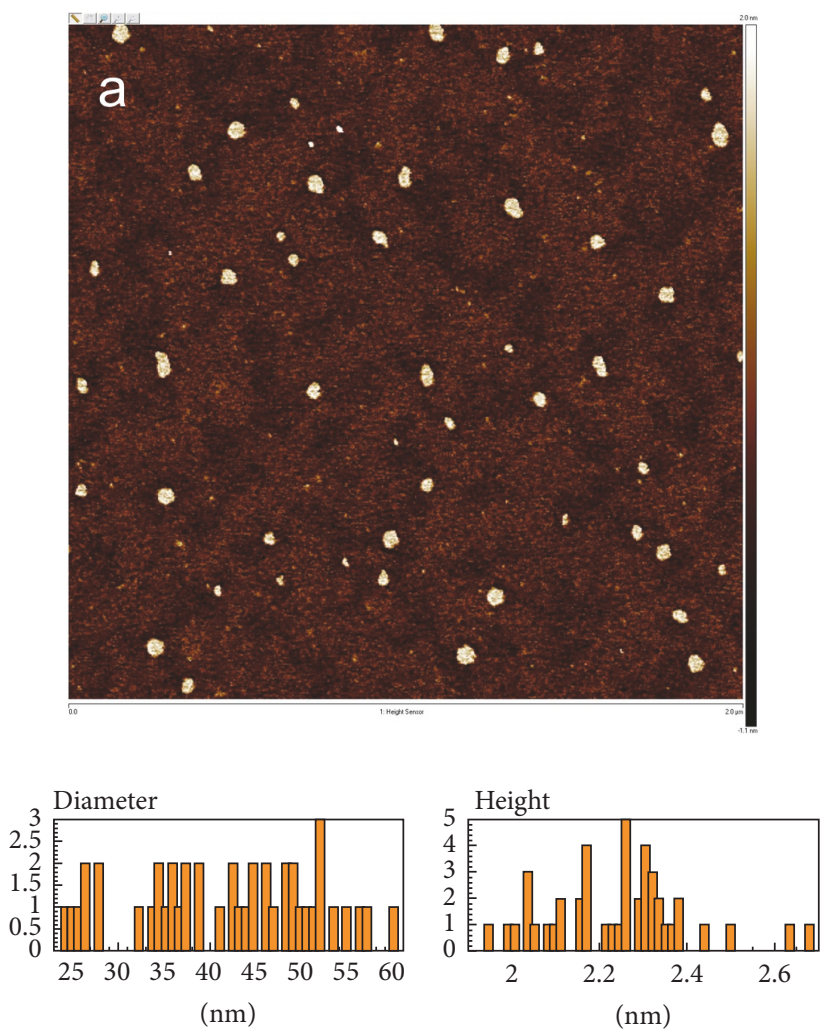

(a)
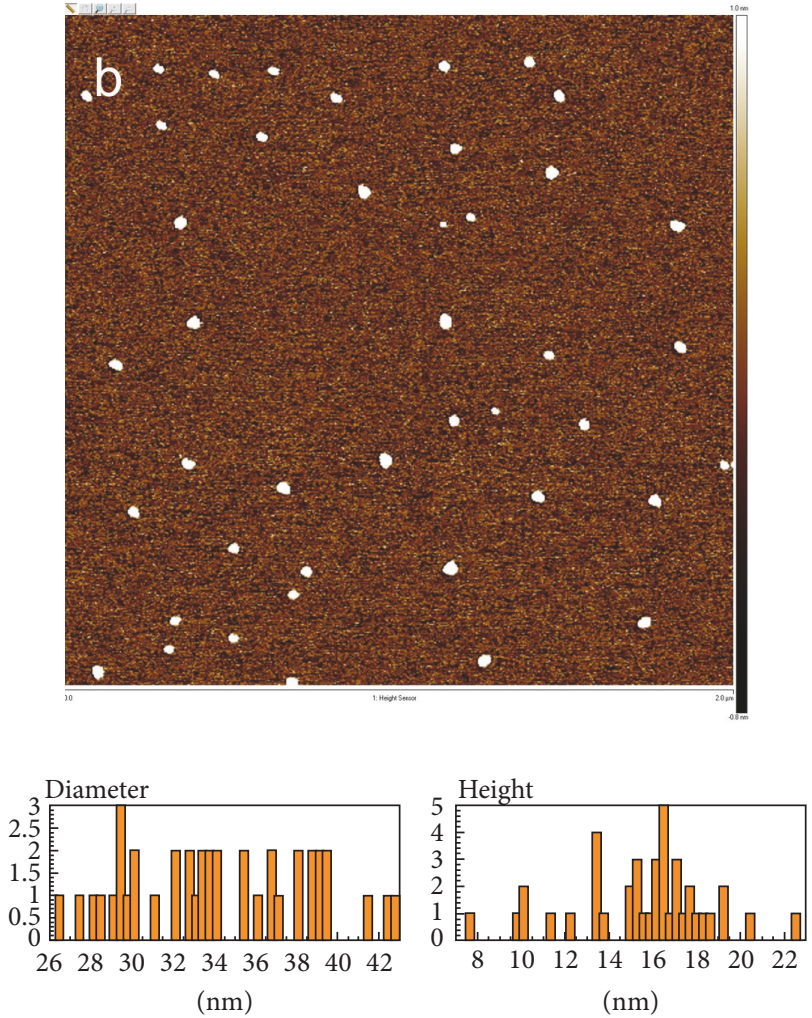

(b)

FIGURE 10: AFM topography of TiQDTs prepared in (a) ethylene glycol and (b) dodecanethiol.

and crystalline areas, and the interlayer spacing was calculated into $0.19 \mathrm{~nm}$ and $0.29 \mathrm{~nm}$ (121), which correspond to brookite (PDF 29-1360), metastable modification of TiO2, and Figure 9(c) presented mapping of elements again. Titania QDTs prepared in ethylene glycol and dodecanethiol, respectively, were used for AFM investigation, and results are showed in Figure 10. In the insets of figures particle size analysis is shown (height profiles, lateral diameters), where it is shown that dots consist preferably of core crystallite height $<2.3 \mathrm{~nm}$ for ethylene glycol and $<16.4 \mathrm{~nm}$ for dodecanethiol. Size distribution of dots is not too wide, but a certain degree of polydispersity is due to partial agglomeration of quantum dot at samples for measurement preparation.

Obtained photoluminescence spectra are shown in Figure 11. The prepared titania quantum dots were excited at different wavelengths ranging from 365 to $405 \mathrm{~nm}$, wavelengths over $405 \mathrm{~nm}$ (ethylene glycol, octadecene, and DMSO) and over $395 \mathrm{~nm}$ (dodecanethiol, DMF, TEOS) already nonexcited luminescence. The increasing wavelength of the excitation light changes the emission maxima of the dispersion, emission maxima observed to vary only between 450 and $500 \mathrm{~nm}$. Emission peaks of quantum dots prepared with top-down method from graphene oxide [14] and ultrasound and exfoliated MoS2 [15] and h-BN and h-BCN [23], respectively, have the much wider range $(400-600 \mathrm{~nm})$, indicating their larger polydispersity. Monodispersed character of TiQDTs is the result of bottom-up method, which leads to an increase in cover dots due to temperature or reaction time.

\section{Conclusions}

Titanium oxides QDTs were prepared with very simple, fast, and cheap bottom-up method. The quantum dots core nuclei precursors were prepared by reaction of titanium oxosulphate and hydrazine hydrate. Formation of quantum dots will never be over $15 \mathrm{~min}$ of a reaction of these nuclei with a suitable solvating agent (ethylene glycol, dimethyl sulfoxide, octadecene, dodecanethiol, dimethylformamide, and tetraethyl orthosilicate). The prepared Ti QDTs are the characteristic narrow range of UV excitation $(365-400 \mathrm{~nm})$ and also a narrow range of emission maxima $(450-500 \mathrm{~nm})$. Given the characteristics of $\mathrm{TiO} 2$, which is nontoxic and biocompatible, one can assume the use of $\mathrm{TiO} 2$ quantum dots in biological applications.

\section{Conflicts of Interest}

The authors declare that they have no conflicts of interest. 

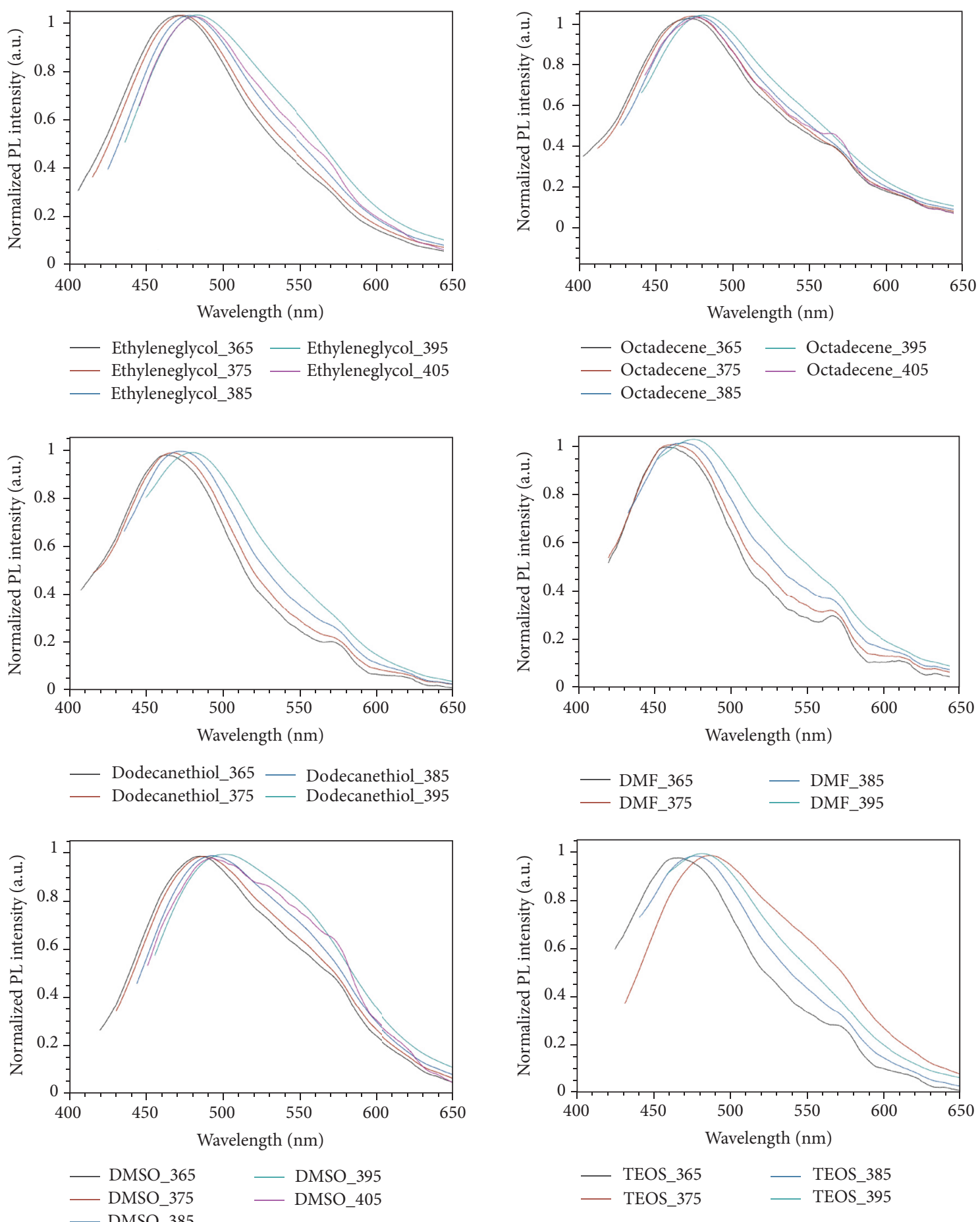

FIGURE 11: PL spectra of TiQDTs prepared in different solvating agents.

\section{Acknowledgments}

This work was supported by RVO 61388980 and Ministry of Industry and Trade of the Czech Republic, no. FV10480.

\section{References}

[1] V. Stengl, I. Netikova, L. Petruzelka, and L. Osterlund, "Detection of DNA Damage Induced by Carbon Nanostructures," in Proceedings of the in Breast Carcinoma Cells in. Fusion
Conference Exploring DNA Repair Pathways as Targets for Cancer Therapy, Cancun, Mexico, 2015.

[2] L. Ahlinder, J. Henych, S. W. Lindström, B. Ekstrand-Hammarström, V. Stengl, and L. Österlund, "Graphene oxide nanoparticle attachment and its toxicity on living lung epithelial cells," RSC Advances, vol. 73, no. 5, pp. 59447-59457, 2015.

[3] Y. Chong, Y. Ma, H. Shen et al., "The in vitro and in vivo toxicity of graphene quantum dots," Biomaterials, vol. 35, no. 19, pp. 5041-5048, 2014. 
[4] L. Ahlinder, B. Ekstrand-Hammarström, P. Geladi, and L. Österlund, "Large uptake of titania and iron oxide nanoparticles in the nucleus of lung epithelial cells as measured by Raman imaging and multivariate classification," Biophysical Journal, vol. 105, no. 2, pp. 310-319, 2013.

[5] H. Peng, J. Li, S.-S. Li, and J.-B. Xia, "First-principles study on rutile TiO2 quantum dots," Journal of Physical Chemistry C, vol. 112, no. 36, pp. 13964-13969, 2008.

[6] J. Drbohlavova, M. Vorozhtsova, R. Hrdy, R. Kizek, O. Salyk, and J. Hubalek, "Self-ordered TiO 2 quantum dot array prepared via anodic oxidation," Nanoscale Research Letters, vol. 7, article no. 123, 2012.

[7] R. Mo, Z. Lei, K. Sun, and D. Rooney, "Facile synthesis of anatase $\mathrm{TiO} 2$ quantum-dot/graphene-nanosheet composites with enhanced electrochemical performance for lithium-ion batteries," Advanced Materials, vol. 26, no. 13, pp. 2084-2088, 2014.

[8] L. Gnanasekaran, R. Hemamalini, and K. Ravichandran, "Synthesis and characterization of $\mathrm{TiO} 2$ quantum dots for photocatalytic application," Journal of Saudi Chemical Society, vol. 19, no. 5, pp. 589-594, 2015.

[9] S. Sood, S. Kumar, A. Umar, A. Kaur, S. K. Mehta, and S. K. Kansal, "TiO2 quantum dots for the photocatalytic degradation of indigo carmine dye," Journal of Alloys and Compounds, vol. 650, pp. 193-198, 2015.

[10] Z. Tachan, I. Hod, M. Shalom, L. Grinis, and A. Zaban, "The importance of the $\mathrm{TiO} 2 /$ quantum dots interface in the recombination processes of quantum dot sensitized solar cells," Physical Chemistry Chemical Physics, vol. 15, no. 11, pp. 38413845, 2013.

[11] V. Štengl, S. Bakardjieva, J. Henych, K. Lang, and M. Kormunda, "Blue and green luminescence of reduced graphene oxide quantum dots," Carbon, vol. 63, pp. 537-546, 2013.

[12] V. Štengl and J. Henych, "Strongly luminescent monolayered $\mathrm{MoS}_{2}$ prepared by effective ultrasound exfoliation," Nanoscale, vol. 5, no. 8, pp. 3387-3394, 2013.

[13] V. Štengl, J. Henych, M. Slušná, and P. Ecorchard, "Ultrasound exfoliation of inorganic analogues of graphene," Nanoscale Research Letters, vol. 9, no. 1, pp. 1-14, 2014.

[14] JCPDS, PDF 2 database, Release 50, International Centre for Diffraction Data, Newtown Square, (2000).

[15] C. Oviedo, "Oxidation kinetics of pure titanium at low pressures," Journal of Physics: Condensed Matter, vol. 5, no. 33 A, article no. 037, pp. A153-A154, 1993.

[16] T. Godfroid, R. Gouttebaron, J. P. Dauchot, P. Leclère, R. Lazzaroni, and M. Hecq, "Growth of ultrathin Ti films deposited on SnO2 by magnetron sputtering," Thin Solid Films, vol. 437, no. 1-2, pp. 57-62, 2003.

[17] K.-Y. Yang, K.-Z. Fung, and M.-C. Wang, "X-ray photoelectron spectroscopic and secondary ion mass spectroscopic examinations of metallic-lithium-activated donor doping process on la 0.56Li0.33TiO3 surface at room temperature," Journal of Applied Physics, vol. 100, no. 5, Article ID 056102, 2006.

[18] A. Sandell, M. P. Andersson, M. K.-J. Johansson et al., "Metalorganic chemical vapor deposition of anatase titanium dioxide on Si: Modifying the interface by pre-oxidation," Surface Science, vol. 530, no. 1-2, pp. 63-70, 2003.

[19] M. V. Kuznetsov, J. F. Zhuravlev, and V. A. Gubanov, "XPS analysis of adsorption of oxygen molecules on the surface of $\mathrm{Ti}$ and TiNx films in vacuum," Journal of Electron Spectroscopy and Related Phenomena, vol. 58, no. 3, pp. 169-176, 1992.
[20] N. Kruse and S. Chenakin, "XPS characterization of $\mathrm{Au} / \mathrm{TiO}_{2}$ catalysts: binding energy assessment and irradiation effects," Applied Catalysis A: General, vol. 391, no. 1, pp. 367-376, 2011.

[21] J. L. Labár, "Consistent indexing of a (set of) single crystal SAED pattern(s) with the ProcessDiffraction program," Ultramicroscopy, vol. 103, no. 3, pp. 237-249, 2005.

[22] S. Xu, D. Li, and P. Wu, "One-pot, facile, and versatile synthesis of monolayer MoS2 /WS2 quantum dots as bioimaging probes and efficient electrocatalysts for hydrogen evolution reaction," Advanced Functional Materials, vol. 25, no. 7, pp. 1127-1136, 2015.

[23] V. Štengl, J. Henych, and M. Kormunda, "Self-assembled BN and BCN quantum dots obtained from high intensity ultrasound exfoliated nanosheets," Science of Advanced Materials, vol. 6, no. 6, pp. 1106-1116, 2014. 

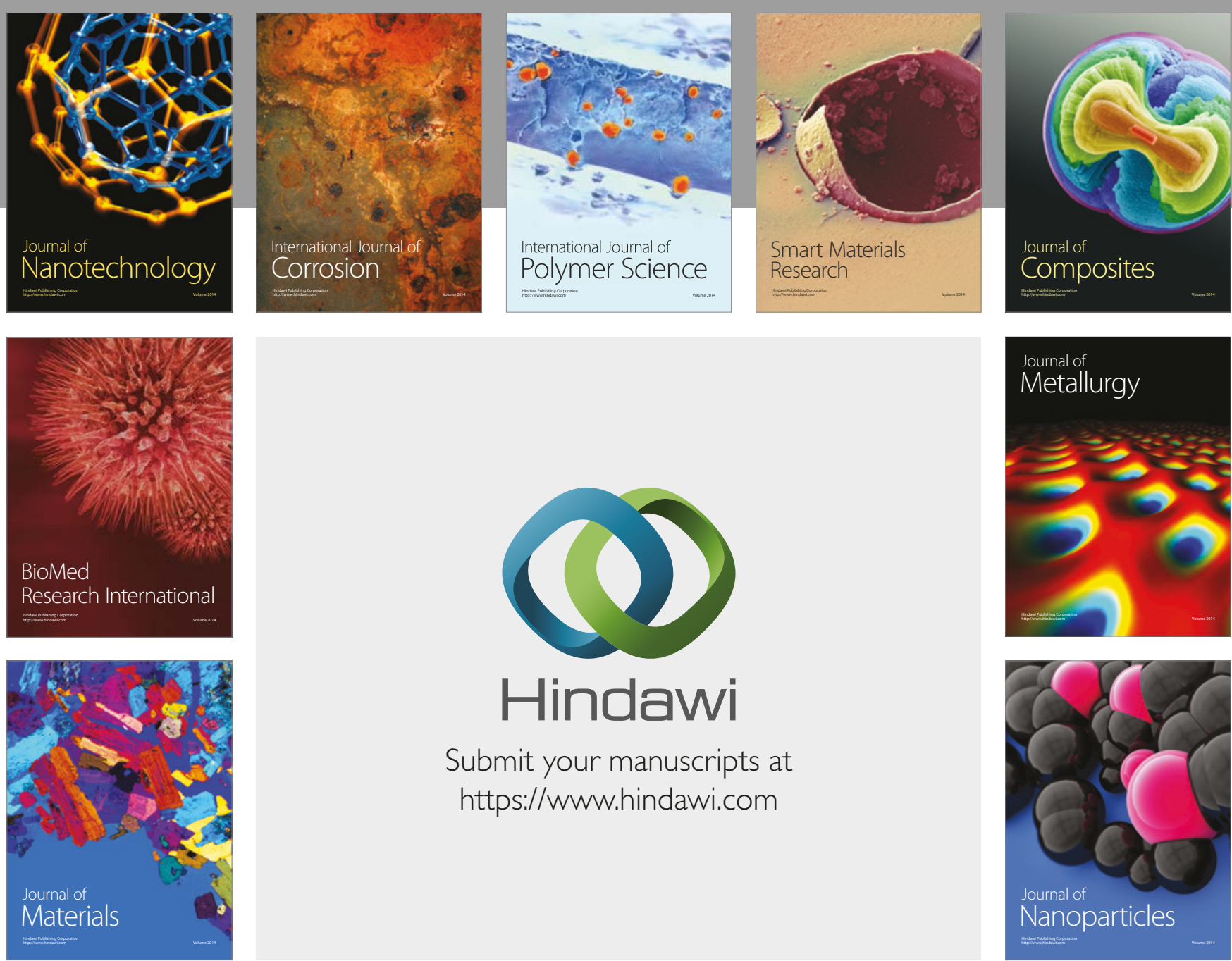

\section{Hindawi}

Submit your manuscripts at

https://www.hindawi.com
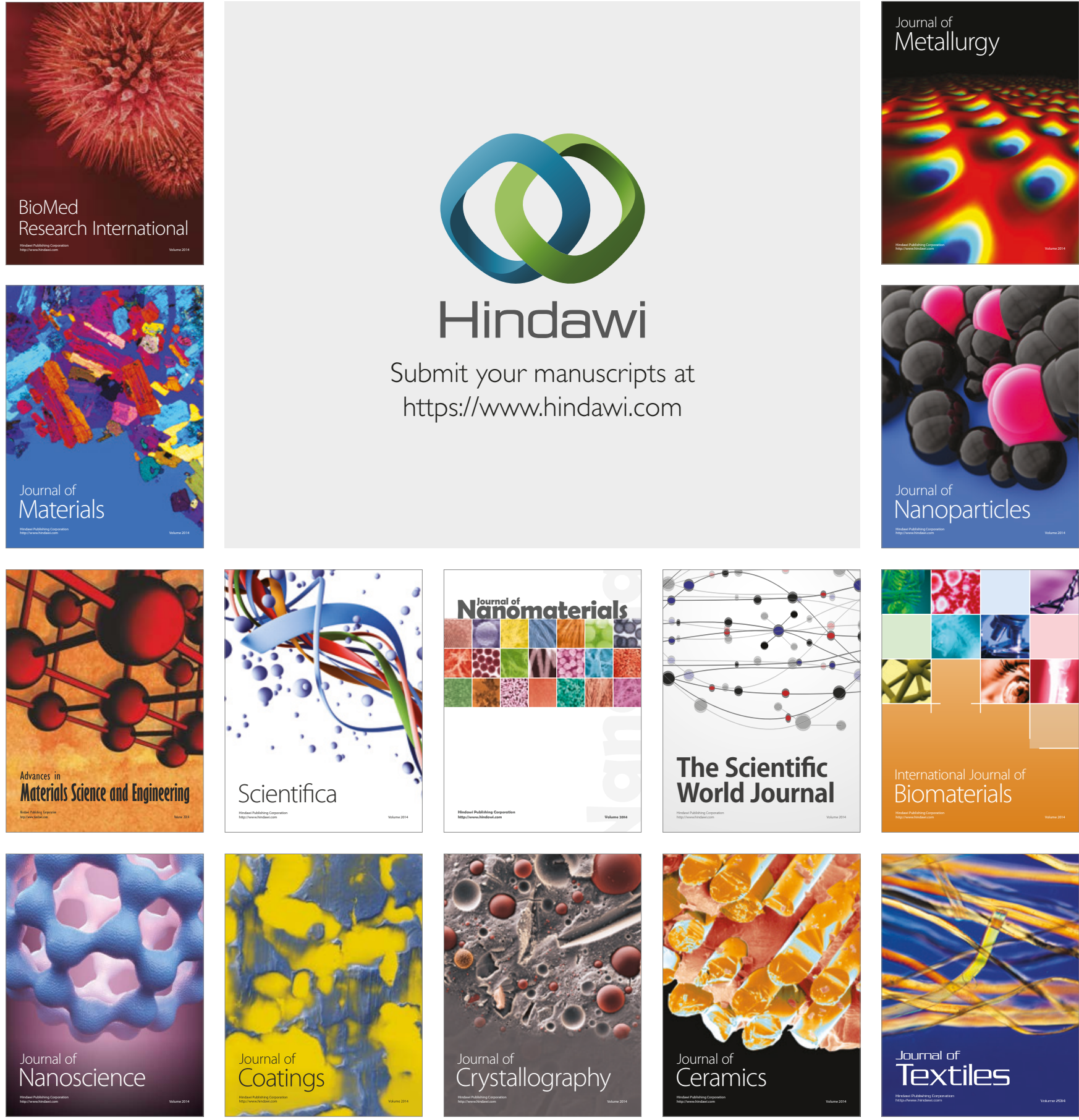

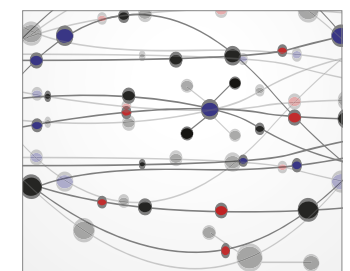

The Scientific World Journal
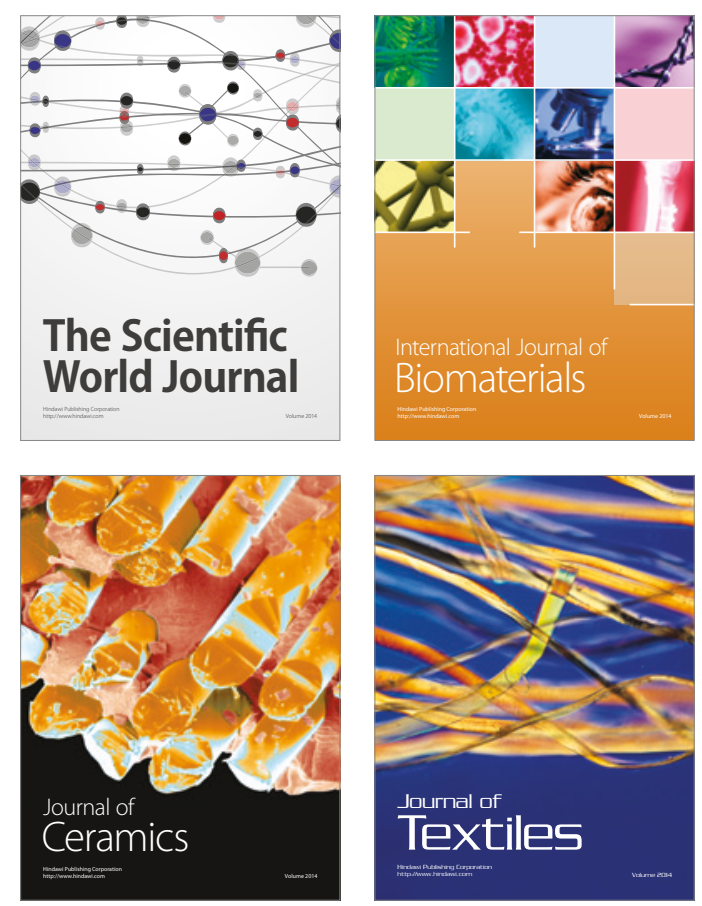